\title{
Le paludisme importé dans la région de Marrakech-Safi, Maroc, entre 1996 et 2016
}

\author{
Imported Malaria in the Region of Marrakech-Safi, Morocco, between 1996 and 2016
}

\author{
Y. El Guamri · O. Amahmid · K. Zenjari - S. Bouhout $\cdot$ M. Ait Mouh $\cdot$ A. Ait Melloul $\cdot$ F. Boraam $\cdot$ H. Benfaida $\cdot$ \\ Kh. Bouhoum · D. Belghyti
}

Reçu le 1 novembre 2017; accepté le 23 février 2018

(C) Société de pathologie exotique et Lavoisier SAS 2018

Résumé En parallèle à l'élimination du paludisme autochtone depuis 2005 et à la certification du Maroc comme pays exempt du paludisme par l'Organisation mondiale de la santé en 2010, des cas de paludisme importés sont encore notifiés. Le présent travail est une étude rétrospective des cas du palu-

Y. El Guamri $(\bowtie) \cdot$ O. Amahmid

Unité de biologie, département des sciences de la vie et de la terre, Centre régional des métiers de l'éducation et de la formation (CRMEF), Marrakech BP 797, Maroc

e-mail : elguamri3000@yahoo.fr

\section{Y. El Guamri · D. Belghyti}

Laboratoire de biotechnologie, environnement et qualité, département de biologie, faculté des sciences, université Ibn-Tofaïl, Kénitra, Maroc

O. Amahmid $\cdot$ Kh. Bouhoum

Laboratoire d'hydrobiologie, écotoxicologie et assainissement, faculté des sciences Semlalia, université Cadi-Ayyad, Marrakech, Maroc

\section{K. Zenjari}

Direction régionale de la santé de Marrakech-Safi, Maroc

\section{S. Bouhout}

Service des maladies parasitaires,

direction de l'épidémiologie et de lutte contre les maladies, ministère de la Santé, Rabat, Maroc

\section{Ait Mouh · F. Boraam}

Service de santé publique et surveillance épidémiologique, Direction régionale de la santé de la région de Marrakech-Safi, Maroc

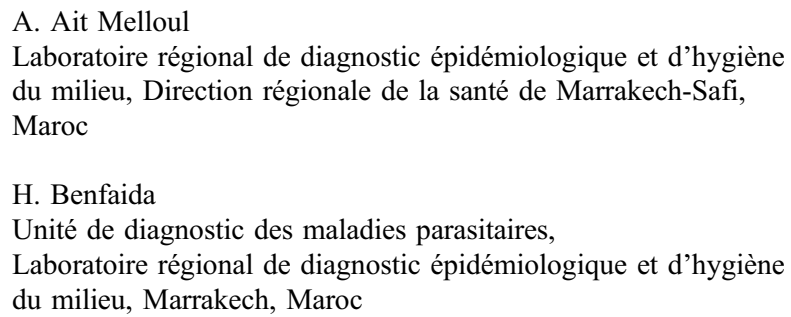

disme d'importation enregistrés au laboratoire de santé publique de Marrakech de 1996 à 2016. Parmi les 208 cas positifs, $89 \%$ sont des hommes (185 patients), soit un sexratio de 8 . Les cas sont importés de 24 pays d'Afrique subsaharienne, en majorité la Guinée équatoriale ( $28 \%$ ), la Guinée Conakry (15\%), la Côte-d'Ivoire (9\%), le Burkina Faso $(8 \%)$ et le Mali (7\%). Les années de plus forte incidence ont été 2012 et 2014 avec 32 cas. Les espèces trouvées sont Plasmodium falciparum dans 177 cas ( $85 \%$ ) et Plasmodium ovale chez 25 malades (12\%). Les autres espèces en cause étaient Plasmodium malariae dans trois cas et Plasmodium vivax dans deux cas. Le nombre de cas a récemment augmenté, probablement lié à l'accroissement des voyages internationaux et des flux migratoires à partir des pays où cette maladie sévit de façon endémique. L'élimination du paludisme autochtone au Maroc et la bonne gestion de cas importés relèvent d'une stratégie nationale de lutte structurée et bien adaptée, axée sur le dépistage, le traitement et la lutte contre les anophèles.

Mots clés Élimination · Paludisme importé · Guinée équatoriale · Guinée Conakry · Côte-d'Ivoire · Burkina Faso · Mali - Plasmodium falciparum - Plasmodium ovale . Marrakech-Safi $\cdot$ Maroc $\cdot$ Afrique du Nord

\footnotetext{
Abstract Since the elimination of the autochthonous malaria in Morocco in 2005, the control of imported malaria, based on epidemiological monitoring of the parasite carriers and on vector control, is a priority. This retrospective study is focused on imported malaria cases identified by optical microscopy at the Laboratory of Public Health in Marrakesh, Morocco, from 1996 to 2016. 208 cases were observed. Males accounted for $89 \%$ of cases. The cases were imported from 24 African countries, especially from Equatorial Guinea (28\%), Guinea Conakry (11\%), Ivory Coast (9\%), Burkina Faso (8\%) and Mali (7\%). The highest incidence was recorded in 2012 and 2014 with 32 cases each. Plasmodium falciparum was the
} 
most frequent parasite ( $85 \%$ ) followed by Plasmodium ovale $(12 \%)$, while lower rates were detected for Plasmodium malariae ( 3 cases) and Plasmodium vivax (2 cases). Increasing malaria cases have been recorded since 1996. This may be related to Morocco's openness to the sub-Saharan Africa with an increase in international travels and migration flow from malaria endemic countries. To keep the status of autochthonous malaria free country, since 2011 the Ministry of Health has developed and implemented a strategy adapted to Moroccan context, to maintain malaria elimination and prevent its reintroduction.

Keywords Elimination - Imported malaria cases ·

Equatorial Guinea · Guinea-Conakry · Côte-d'Ivoire ·

Burkina Faso - Mali · Plasmodium falciparum ·

Plasmodium ovale $\cdot$ Marrakech-Safi $\cdot$ Morocco $\cdot$ Northern Africa

\section{Introduction}

Depuis 2005, aucun cas de paludisme autochtone n'a été dépisté au Maroc [6]. Les cas de paludisme d'importation sont généralement localisés dans les grandes agglomérations urbaines où le risque de transmission est pratiquement nul. La majorité des personnes concernées sont localisables et encadrées avant leur départ et à leur arrivée, particulièrement celles qui voyagent dans le cadre de la coopération Sud-Sud [13]. La région de Marrakech-Safi est exposée au risque de réintroduction du paludisme à cause du mouvement de la population qu'elle subit, du nombre de migrants vivant au Maroc et retournant dans leur pays d'origine et des voyageurs marocains occasionnels en Afrique subsaharienne. Cette région est considérée comme ayant un passé épidémiologique, puisque aucun cas de paludisme autochtone n'y a été enregistré depuis plus de 15 ans [3]. Elle présente une certaine vulnérabilité due à l'existence d'une population anophélienne vectrice dans des zones où les conditions seraient favorables à la reprise de la transmission [3,12].

La ville de Marrakech, métropole de la région de Marrakech-Safi en tant que ville universitaire et capitale touristique, est un pôle d'attraction pour les ressortissants de nombreux pays d'Afrique subsaharienne. L'importation des cas de paludisme est une réalité à intégrer dans la stratégie de lutte antipaludique (LAP). L'élimination des cas autochtones ne doit pas signifier l'abandon de la lutte, mais le renforcement du dispositif de veille et de vigilance ainsi que des mesures de prophylaxie des voyageurs qui se déplacent dans les zones endémiques. La stratégie nationale post-certification de lutte contre le paludisme a pour objectifs majeurs l'éviction des complications et de la mortalité liée au paludisme importé et la prévention de la réintroduction du paludisme au Maroc.
Ce travail présente la situation, l'évolution et les caractéristiques épidémiologiques des cas positifs de paludisme importé enregistrés au niveau du laboratoire de santé publique (LSP) de la Direction régionale de la Santé de Marrakech-Safi de 1996 à 2016.

\section{Patients et méthodes}

\section{Zone d'étude}

L'étude a été réalisée au niveau de l'unité des maladies parasitaires du LSP de la Direction régionale de la Santé de Marrakech-Safi, seule entité du ministère de la Santé opérant sur le diagnostic du paludisme et sur le contrôle de cette maladie au niveau régional.

\section{Période et durée de l'étude}

Nous rapportons une étude rétrospective descriptive portant sur les cas de paludisme importé diagnostiqués au LSP et traités soit en ambulatoire au niveau du service des réseaux des établissements de santé, soit en milieu hospitalier à Marrakech, durant la période comprise entre le $1^{\text {er }}$ janvier 1996 et le 31 décembre 2016. Les étalements sanguins examinés provenaient de différents centres médicaux de la région de Marrakech, y compris de patients suspects présentant une fièvre (ouvriers, migrants, touristes, étudiants ou étrangers en formation, militaires).

\section{Diagnostic et analyse parasitologique}

Le diagnostic de paludisme était microscopique [10]. L'âge, le sexe, la profession, la nationalité, le pays visité, l'espèce plasmodiale, la prise de chimioprophylaxie, le délai entre le retour du pays d'endémie et la consultation, le traitement suivi et l'évolution sous traitement ont été notés. Les cas positifs ont été pris en charge par le service de médecine interne et au service de réanimation pour les cas compliqués.

\section{Résultats}

Au cours de la période 1996 à 2016, 208 cas ont été trouvés positifs, avec une nette prédominance masculine (185 hommes et 23 femmes), soit un sex-ratio de 8. L'âge moyen était de $32 \pm 5$ ans, avec des extrêmes de 2 et 70 ans. La tranche d'âge la plus touchée était celle comprise entre 21 et 30 ans. Aucun cas d'enfant âgé de moins de deux ans n'a été notifié (Fig. 1). Le nombre de cas a nettement augmenté à partir de 2010 (Fig. 2). Plasmodium falciparum est de loin l'espèce la plus prévalente. Plasmodium malariae a été détecté en 2012, 
2014 et 2016 et Plasmodium vivax en 2000 et 2015. $P$. falciparum a été l'hématozoaire observé chez 177 cas, soit $85 \%$, suivi de Plasmodium ovale (26 cas, soit $12 \%$ ). Les autres espèces en cause étaient $P$. vivax (deux cas) et P. malariae (trois cas). En 20 ans, six décès ont été rapportés (un en 2006 et en 2009, deux décès en 2012 et en 2014).
Les pays concernés par le paludisme importé sont au nombre de 14 et appartiennent à différentes régions (Afrique, Asie, Europe, etc.) (Tableau 1).

Les principaux pays de contamination sont d'Afrique, plus particulièrement l'Afrique de l'Ouest avec 173 cas. Par pays, les nombres de cas les plus élevés concernent la

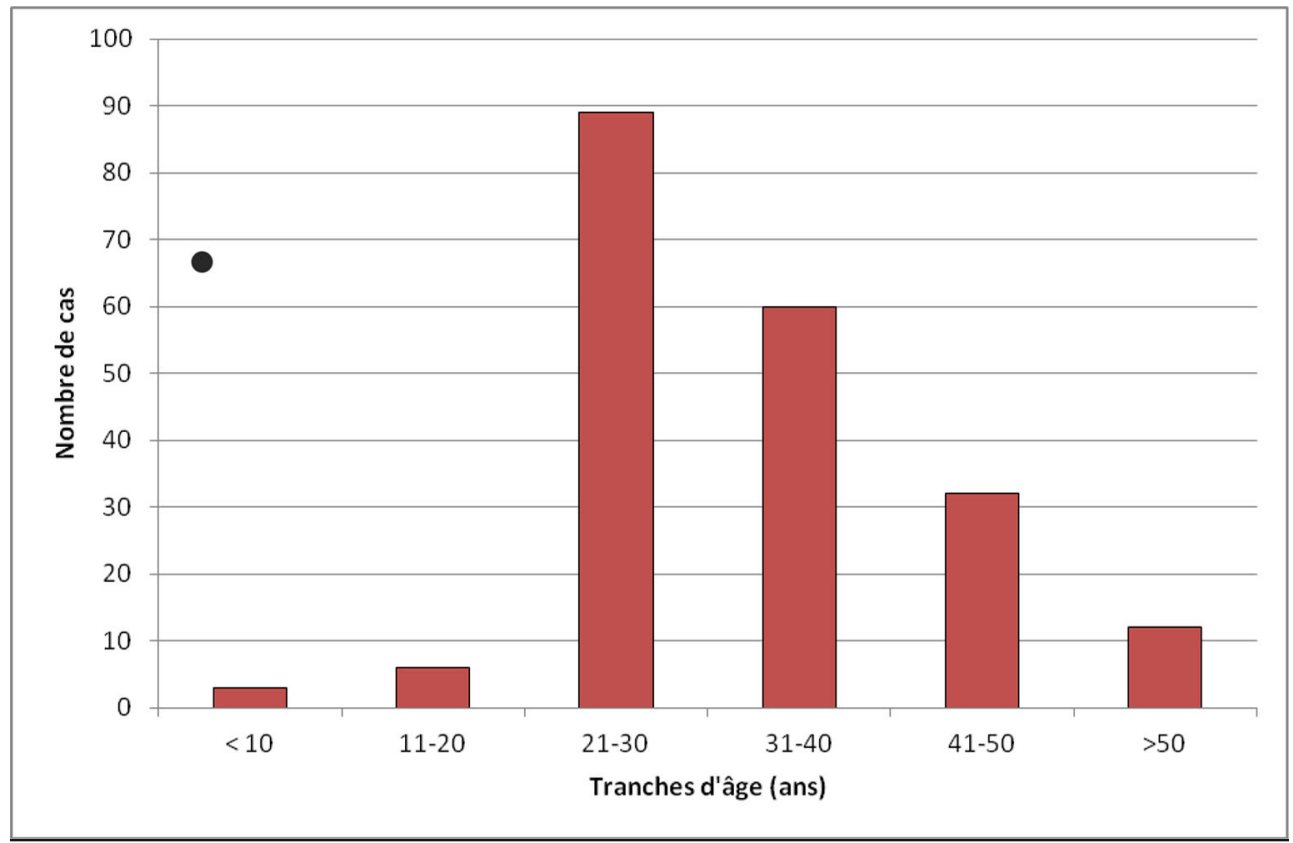

Fig. 1 Répartition des cas positifs du paludisme importé en fonction de l'âge / Distribution of imported malaria patients by age group

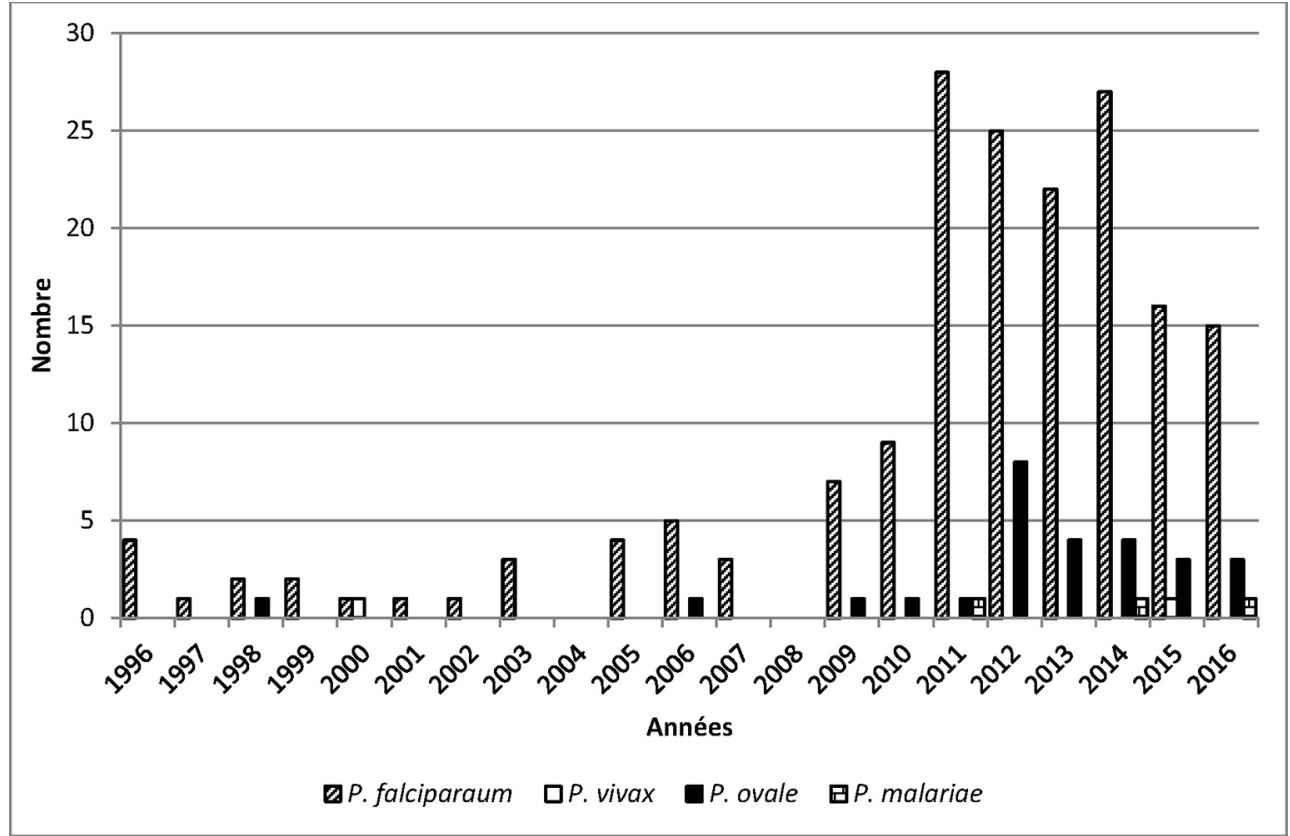

Fig. 2 Évolution annuelle des cas de paludisme importés selon les espèces plasmodiales identifiées / Annual evolution of imported malaria cases by causal plasmodial species 
Tableau 1 Répartition des cas importés selon la nationalité des patients et l'espèce plasmodiale dans la région Marrakech-Safi, Maroc, 1996-2016 / Distribution imported malaria cases by origin and plasmodial species (Marrakech-Safi, Morocco, 1996-2016)

\begin{tabular}{|c|c|c|c|c|c|c|}
\hline \multirow[t]{2}{*}{ Origine/Région } & \multicolumn{6}{|c|}{ Espèces plasmodiales } \\
\hline & P. falciparum & P. vivax & P. ovale & P. malariae & Associées & Total \\
\hline Afrique de l'Ouest & 45 & 1 & 4 & & & 50 \\
\hline Afrique de l'Est & 1 & & & & & 1 \\
\hline Afrique centrale & 2 & & & & & 2 \\
\hline Europe & 7 & & & & & 7 \\
\hline Amérique latine & 1 & & & & & 1 \\
\hline Asie & 1 & & & & & 1 \\
\hline Australie & & & 1 & & & 1 \\
\hline Maroc & 120 & 1 & 21 & 3 & & 145 \\
\hline Total & 177 & 2 & 26 & 3 & $\mathbf{0}$ & 208 \\
\hline
\end{tabular}

Guinée équatoriale (60 cas), la Guinée Conakry (32 cas), la Côte-d'Ivoire (20 cas). Les malades ont pour $94 \%$ d'entre eux séjourné dans un seul pays endémique (Tableaux 2, 3).

\section{Chimioprophylaxie et traitement antipaludique des cas de paludisme importés de l'étranger}

La chloroquine (Nivaquine ${ }^{\circledR}$ ) et l'artéméther-luméfantrine $\left(\right.$ Coartem $\left.^{\circledR}\right)$ ont été les plus utilisés pour la prophylaxie et le traitement thérapeutique des cas de paludisme importé.

\section{Discussion}

Nos résultats en termes d'âge et de sexe des patients sont comparables à ceux rapportés dans les données épidémiologiques nationales de la direction d'épidémiologie et de lutte contre les maladies (DELM) au Maroc [9,11,12], dans lesquelles les cas de notre série sont inclus.

Le paludisme d'importation est un problème qui se pose avec acuité en raison du développement des voyages inter- nationaux, du tourisme et des migrations internationales [13]. Le nombre de personnes voyageant entre le Maroc et les pays d'endémie palustre (particulièrement en Afrique) a augmenté au cours de ces dernières années, avec un taux de croissance de 12 \% rapporté pour l'année 2016 [14]. Dans

Tableau 3 Répartition des cas de paludisme importé de l'étranger selon le nombre de pays de séjour (Marrakech-Safi, Maroc, 1996-2016) / Distribution of imported malaria cases according to the number of countries of stay (Marrakech-Safi, Morocco, 1996-2016)

\begin{tabular}{|ll|}
\hline & $\begin{array}{l}\text { Nombre de cas } \\
\text { de paludisme importé }\end{array}$ \\
\hline 1 seul pays de séjour & 178 \\
2 pays de séjour & 6 \\
3 pays de séjour & 12 \\
4 pays de séjour & 1 \\
Pays de séjour non précisé & 11 \\
Total & $\mathbf{2 0 8}$ \\
\hline
\end{tabular}

Tableau 2 Répartition des cas importés dans la région Marrakech-Safi, Maroc, selon le pays de séjour des patients et l'espèce plasmodiale, entre 1996 et 2016 / Distribution of imported malaria cases in Marrakech-Safi, Morocco, by country of stay and plasmodial species (1996-2016)

\begin{tabular}{|llllll|}
\hline Région de séjour & P. falciparum & P. ovale & P. vivax & P. malariae & Total \\
\hline Afrique de l'Ouest & 147 & 21 & 2 & 2 & 173 \\
Afrique de l'Est & 2 & 1 & & & 3 \\
Afrique centrale & 12 & 2 & & & 14 \\
Afrique de l'Est-Amérique latine & & 1 & & 1 \\
Afrique de l'Ouest et centrale & 1 & & 1 & 1 \\
$\begin{array}{l}\text { Afrique de l'Ouest-Afrique de l'Est-cône sud } \\
\text { Asie }\end{array}$ & 1 & & & 1 \\
Non précisée & 14 & & & 13 \\
Total & $\mathbf{1 7 7}$ & $\mathbf{2 6}$ & $\mathbf{2}$ & $\mathbf{3}$ & $\mathbf{2 0 8}$ \\
\hline
\end{tabular}


les pays qui ont éliminé le paludisme, les cas de paludisme importé sont diagnostiqués en majorité chez des voyageurs adultes. Les populations concernées sont surtout les voyageurs marocains occasionnels en Afrique subsaharienne ainsi que les militaires marocains déployés dans le cadre des missions de maintien de la paix en Afrique, les expatriés et les migrants vivant au Maroc [5,7,8]. Dans notre cas, il s'agit essentiellement des ouvriers, des fonctionnaires et des étudiants marocains résidant en zone d'endémie palustre. La Guinée Conakry, la Guinée équatoriale, le Gabon, le Mali, la Côte-d'Ivoire et la Mauritanie sont les pays les plus visités et les plus incriminés dans notre étude. Ce qui rejoint les résultats de l'étude de Bouratbine et al. [4] rapportant que 92,7\% des sujets étaient originaires ou avaient séjourné en Afrique subsaharienne. Un taux de $98,3 \%$ a été cité dans l'étude d'Ayadi et al. [2]. La problématique de cas importés avait commencé à se poser à partir de la fin des années 1970, suite à l'accroissement des mouvements de ressortissants marocains vers les pays d'Afrique subsaharienne et des effectifs croissants d'étudiants africains au Maroc.

Par ailleurs, le risque de la réintroduction du paludisme ne peut être écarté en raison de la persistance de la présence des vecteurs [13]. Le Programme national de lutte contre le paludisme a permis de contrôler la transmission de cette maladie, le nombre de cas de paludisme étant passé d'environ 30000 en 1965 à une centaine de cas à la fin des années 1990. Aucun cas local n'a été signalé depuis 2005, permettant la certification d'exemption de paludisme par l'Organisation mondiale de la santé (OMS) en 2010 [6]. Des cas d'importation sont enregistrés chaque année [1]. Leur nombre a connu une augmentation à partir de l'année 2010. Celle-ci serait due à l'accroissement d'échanges, de voyages et des flux migratoires des pays où la maladie sévit de façon endémique, et aux mesures de contrôle du paludisme importé mises en place. En effet, le ministère de la Santé met en œuvre, depuis 2012, des mesures de prévention concernant principalement le diagnostic et la prise en charge rapide et gratuite de tous les cas importés, le renforcement des mesures de surveillance épidémiologique et de lutte contre les vecteurs ainsi que des actions de prévention pour les voyageurs se rendant dans des pays endémiques. Le maintien de cet acquis nécessite une vigilance continue pour éviter la réémergence de cette maladie au Maroc.

\section{Conclusion}

Le paludisme importé est la seule forme de la maladie notifiée au Maroc et en particulier à Marrakech depuis l'année 2005. À partir de 2010, une augmentation du nombre de cas importés a été notée, avec une prédominance de $P$. falciparum. Il est important de maintenir la dynamique des efforts de prévention, de lutte et d'élimination observée au cours de la décennie écoulée.

Remerciements Les auteurs tiennent à remercier vivement S. Hadhoumi de l'unité de diagnostic des maladies parasitaires du Laboratoire régional de diagnostic épidémiologique et d'hygiène du milieu et $\mathrm{M}$. Essarafy du Programme de lutte contre les maladies parasitaires, du service des réseaux des établissements de santé (SRES) à Marrakech pour leur contribution à la réalisation de ce travail

Liens d'intérêt : Les auteurs déclarent ne pas avoir de liens d'intérêt.

\section{Références}

1. Anouti MA (2017) Le paludisme d'importation : expérience de l'hôpital militaire Avicenne de Marrakech. Thèse de doctorat de médecine. Faculté de médecine et de pharmacie. Université CadiAyyad. Marrakech, $222 \mathrm{p}$

2. Ayadi A, Makni F, Sellam IH, et al (2000) Le paludisme d'importation à Sfax (Tunisie), Med Trop 60:99

3. Barkia A, Laamrani El Idrissi A, Mouki B, et al (2000) Stratégie d'élimination du paludisme au Maroc plan et éléments d'évaluation. Édité avec le soutien de l'Organisation mondiale de la santé. Ministère de la Santé. Maroc, $41 \mathrm{p}$

4. Bouratbine A, Chahed MK, Aoun K, et al (1998) Le paludisme d'importation en Tunisie. Bull Soc Pathol Exot 91:203-7 [http:// www.pathexo.fr/documents/articles-bull/T91-3-1847.pdf]

5. Charraa, B, Sodqib M, Sandalia O, et al (2007) Paludisme grave d'importation chez l'adulte : étude rétrospective de dix cas admis en réanimation à Casablanca. Med Mal Infect 37:162-5. doi: 10.1016/j.medmal.2006.09.006

6. Direction d'épidémiologie et de lutte contre les maladies (DELM) (2010) Bilan des maladies parasitaires. Ministère de la Santé. Rabat, Maroc, $74 \mathrm{p}$

7. El Mehdi S (2015) Les circonstances de découverte du paludisme d'importation : expérience du service de médecine interne de l'hôpital militaire Avicenne de Marrakech. Thèse de médecine. Faculté de médecine et de pharmacie. Université Cadi-Ayyad, $177 \mathrm{p}$

8. El Ouali Lalami A, Cherigui M, Koraichi SI, et al (2009) Le paludisme importé dans le Centre-Nord du Maroc entre 1997 et 2007. Cahiers Santé 19:1

9. Ministère de la Santé (2013) Santé en chiffres. Bilan des maladies parasitaires. DELM, Rabat, 2012 [en ligne]

10. Ministère de la Santé (2014) Protocole national de la prise en charge thérapeutique du paludisme. Guide de prise en charge thérapeutique du paludisme importé, $20 \mathrm{p}$

11. Ministère de la Santé (2014) Santé en chiffres. Bilan des maladies parasitaires. DELM, Rabat, 2013 [en ligne]

12. Ministère de la Santé (2015) Santé en chiffres. Bilan des maladies parasitaires. DELM, Rabat, 2014 [en ligne]

13. OMS (2007) Le paludisme au Maroc — une lutte sans relâche, une perspective d'élimination/Organisation mondiale de la santé. Bureau régional de la Méditerranée orientale, Royaume du Maroc. Ministère de la Santé, $124 \mathrm{p}$

14. ONDA (Office national des aéroports) (2017) Aéroports du Maroc : trafic aérien de l'année 2016. Communiqué de presse $n^{\mathrm{o}} 2 / 17,3 \mathrm{p}$ 